\title{
Engineering fast and stable splitting of matter waves
}

\author{
E. Torrontegui, ${ }^{1, *}$ S. Martínez-Garaot, ${ }^{1}$ M. Modugno, ${ }^{2,3}$ Xi Chen, ${ }^{1,4}$ and J. G. Muga ${ }^{1,4}$ \\ ${ }^{1}$ Departamento de Química Física, Universidad del País Vasco UPV/EHU, Apartado 644, Bilbao, Spain \\ ${ }^{2}$ Departamento de Física Teórica e Historia de la Ciencia, Universidad del País Vasco UPV/EHU, Apartado 644, Bilbao, Spain \\ ${ }^{3}$ IKERBASQUE, Basque Foundation for Science, Alameda Urquijo 36, 48011 Bilbao, Spain \\ ${ }^{4}$ Department of Physics, Shanghai University, 200444 Shanghai, People's Republic of China \\ (Received 13 July 2012; revised manuscript received 1 February 2013; published 28 March 2013)
}

\begin{abstract}
When attempting to split a coherent noninteracting atomic cloud by bifurcating the initial trap into two well-separated wells, slow adiabatic following is unstable with respect to any slight trap asymmetry, and the matter wave "collapses" to the well with the lowest energy. A generic fast chopping splits the wave but it also excites it. Shortcuts to adiabaticity engineered to speed up the unperturbed adiabatic process through nonadiabatic transients provide, instead, quiet and robust balanced splitting. For a Bose-Einstein condensate in the mean-field limit, the interatomic interaction makes the splitting, adiabatic or via shortcuts, more stable with respect to trap asymmetry. Simple formulas are provided to distinguish different regimes.
\end{abstract}

DOI: 10.1103/PhysRevA.87.033630

PACS number(s): $03.75 . \mathrm{Kk}, 37.10 . \mathrm{Gh}, 42.50 . \mathrm{Dv}$

\section{INTRODUCTION}

The splitting of a wave packet is an important operation in matter wave interferometry [1-4]. A strategy to improve the interferometer performance is to suppress the interaction [5,6], so let us first consider a noninteracting Bose-Einstein condensate (BEC). For this system, complete wave splitting into two separated branches is a peculiar operation because adiabatic following, rather than robust, is intrinsically unstable with respect to a small external potential asymmetry [7]. The potential is assumed here to evolve from a single well to a final double-well where tunneling is negligible [8]. The groundstate wave function "collapses" into the final lower well (or, more generally, into the one that holds the lowest ground state as in [7]) and a very slow trap potential bifurcation fails to split the wave except for perfectly symmetrical potentials. A fast bifurcation remedies this but the price is typically a strong excitation, which is also undesired, as it produces loss of contrast in the interference patterns when recombining the two waves [9]. We propose here a way around these problems by using shortcuts to adiabaticity that speed up the adiabatic process along a nonadiabatic route [10]. Wave splitting via shortcuts avoids the final excitation and is significantly more stable with respect to asymmetry than the adiabatic following. Specifically we use a streamlined version [11] of the fastforward (FF) technique of Masuda and Nakamura [12] applied to the Gross-Pitaevskii (GP) or Schrödinger equations. We have previously found some obstacles to application of the invariant-based method (quadratic-in-momentum invariants do not satisfy the required boundary conditions [11]) and the transitionless-driving algorithm [13] (because of difficulties in implementing counter-diabatic terms in practice).

In Sec. II we summarize the FF approach for condensates (interacting or not) in one dimension and its application to splitting. In Sec. III the effect of a small asymmetric perturbation is studied for noninteracting matter waves, and Sec. IV analyzes and interprets the results with the aid of a moving two-mode model. Section V studies the remarkable

*eriktorrontegui@gmail.com stability with respect to the asymmetry achieved due to interatomic interactions in the mean-field limit, and different regimes are distinguished. Finally, Sec. VI discusses the results and open questions.

\section{FAST-FORWARD APPROACH}

The FF method [11,12,14] may be used to generate external potentials $V_{\mathrm{FF}}$ and drive the matter wave from an initial single well to a final symmetric double-well. In one dimension, the starting point of the streamlined version in [11] is the time-dependent GP equation $i \hbar \partial_{t}|\psi(t)\rangle=H(t)|\psi(t)\rangle$, where $H(t)=T+G(t)+V(t)$ includes the kinetic energy $T$, external potential $V$, and mean-field potential $G$. Assuming a local $V,\left\langle x|V(t)| x^{\prime}\right\rangle=V(x, t) \delta\left(x-x^{\prime}\right)$, we formally solve for it in the GP equation,

$V(x, t)=\left[i \hbar\left\langle x \mid \partial_{t} \psi(t)\right\rangle-\langle x|T+G| \psi(t)\rangle\right] /\langle x \mid \psi(t)\rangle$,

with $\langle x|T| \psi(t)\rangle=\frac{-\hbar^{2}}{2 m} \psi^{\prime \prime}(x, t) \quad$ and $\quad\langle x|G(t)| \psi(t)\rangle=g_{1} N$ $|\psi(x, t)|^{2} \psi(x, t)$. The primes denote derivatives with respect to $x, g_{1}$ is the effective, one-dimensional (1D) coupling constant of the BEC, and $N$ is the number of atoms. For the numerical examples we consider ${ }^{87} \mathrm{Rb}$ atoms. Using in Eq. (1) the ansatz $\langle x \mid \psi(t)\rangle=r(x, t) e^{i \phi(x, t)}, r(x, t), \phi(x, t) \in \mathbb{R}$, then

$$
\begin{gathered}
\operatorname{Re}[V(x, t)]=-\hbar \dot{\phi}+\frac{\hbar^{2}}{2 m}\left(\frac{r^{\prime \prime}}{r}-\left(\phi^{\prime}\right)^{2}\right)-g_{1} N r^{2}, \\
\operatorname{Im}[V(x, t)]=\hbar \frac{\dot{r}}{r}+\frac{\hbar^{2}}{2 m}\left(\frac{2 \phi^{\prime} r^{\prime}}{r}+\phi^{\prime \prime}\right),
\end{gathered}
$$

where the overdot indicates the time derivative. We impose $\operatorname{Im}[V(x, t)]=0$ and require that the ground state of the initial Hamiltonian $H(0)$ evolve in a time $t_{f}$ into the corresponding ground state of the final $H\left(t_{f}\right)$, assuming that the Hamiltonian is given at the boundary times. In the inversion protocol, $r(x, t)$ is designed first, and Eq. (3) is solved for $\phi$ to get $V_{\mathrm{FF}}(x, t):=\operatorname{Re}[V(x, t)]$ from Eq. (2). To ensure that the initial and final states are eigenstates of the stationary GP equation we impose $\dot{r}=0$ at $t=0$ and $t_{f}$. Then Eq. (3) has solutions $\phi(x, t)$ independent of $x$ at the boundary times [11]. Using this 

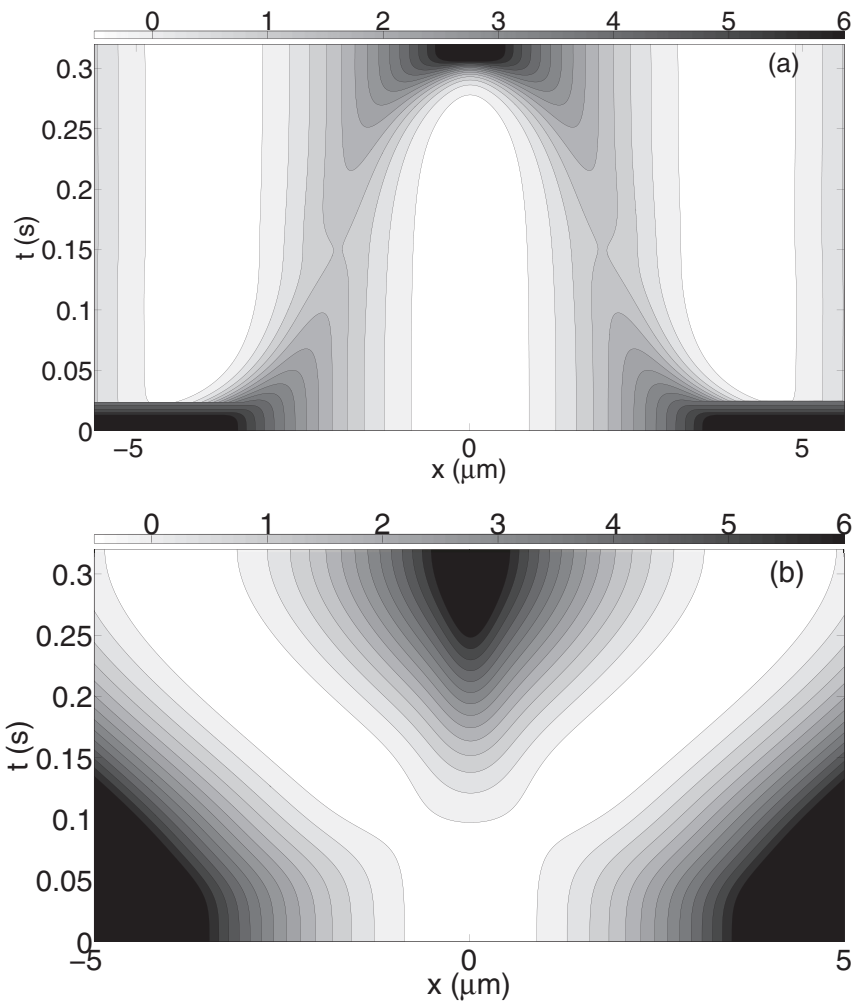

FIG. 1. Contour plot of $V_{\mathrm{FF}}$ in units $\hbar \omega$ from Eq. (2) for (a) a three-well interpolation and (b) a $Y$-shaped form. Parameters: $\omega=780 \mathrm{rad} / \mathrm{s}$, and $t_{f}=320 \mathrm{~ms}$.

in Eq. (2) at $t=0$, and multiplying by $e^{i \phi(0)}$,

$$
\left[\frac{-\hbar^{2}}{2 m} \nabla^{2}+V(x, 0)+g_{1} N|\psi(x, 0)|^{2}\right] \psi(x, 0)=-\hbar \dot{\phi}(0) \psi(x, 0) .
$$

The initial state $\psi(x, 0)$ is an eigenstate of the stationary GP equation with chemical potential $-\hbar \dot{\phi}(0)=\mu(0)$. A similar result is found at $t_{f}$.

In the following two sections we consider first $g_{1}=0$ and split an initial single-Gaussian state $f(x, 0)=e^{-x^{2} / 2 a_{0}^{2}}$ $\left(a_{0}=\sqrt{\hbar / m \omega}\right)$ into a final double-Gaussian $f\left(x, t_{f}\right)=$ $e^{-\left(x-x_{f}\right)^{2} / 2 a_{0}^{2}}+e^{-\left(x+x_{f}\right)^{2} / 2 a_{0}^{2}}$. In previous works $[11,12]$ use has been made of the interpolation $r(x, t)=z(t)\{[1-\mathcal{R}(t)]$ $\left.f(x, 0)+\mathcal{R}(t) f\left(x, t_{f}\right)\right\}$, where $\mathcal{R}(t)$ is a smooth, monotonously increasing function from 0 to 1 , and $z(t)$ is a normalization function. This produces a triple-well potential at intermediate times. Here we use instead the two-bump form $r(x, t)=z(t)\left[e^{-\left[x-x_{0}(t)\right]^{2} / 2 a_{0}^{2}}+e^{-\left[x+x_{0}(t)\right]^{2} / 2 a_{0}^{2}}\right]$, which generates simpler $Y$-shaped potentials (see Fig. 1). We impose $\dot{x}_{0}(0)=\dot{x}_{0}\left(t_{f}\right)=0$, so $\dot{r}=0$ at the boundary times. In the numerical examples $x_{0}(s)=x_{f}\left(3 s^{2}-2 s^{3}\right)$, where $s=t / t_{f}$, and $x_{f}=4 \mu \mathrm{m}$ (see, e.g., [15]); Equation (3) is solved with the initial conditions $\phi(x=0)=\left.\frac{\partial \phi}{\partial x}\right|_{x=0}=0$.

\section{EFFECT OF THE PERTURBATION}

Assume now a perturbed Hamiltonian $H_{\lambda}=T+V_{\lambda}$ with $V_{\lambda}=V_{\mathrm{FF}}+\lambda \theta(x)$, where $\theta(x)$ is the step function and $\lambda$ the potential imbalance. Except in the final discussion, we assume that $\lambda$ is some uncontrollable and hard-to-avoid small
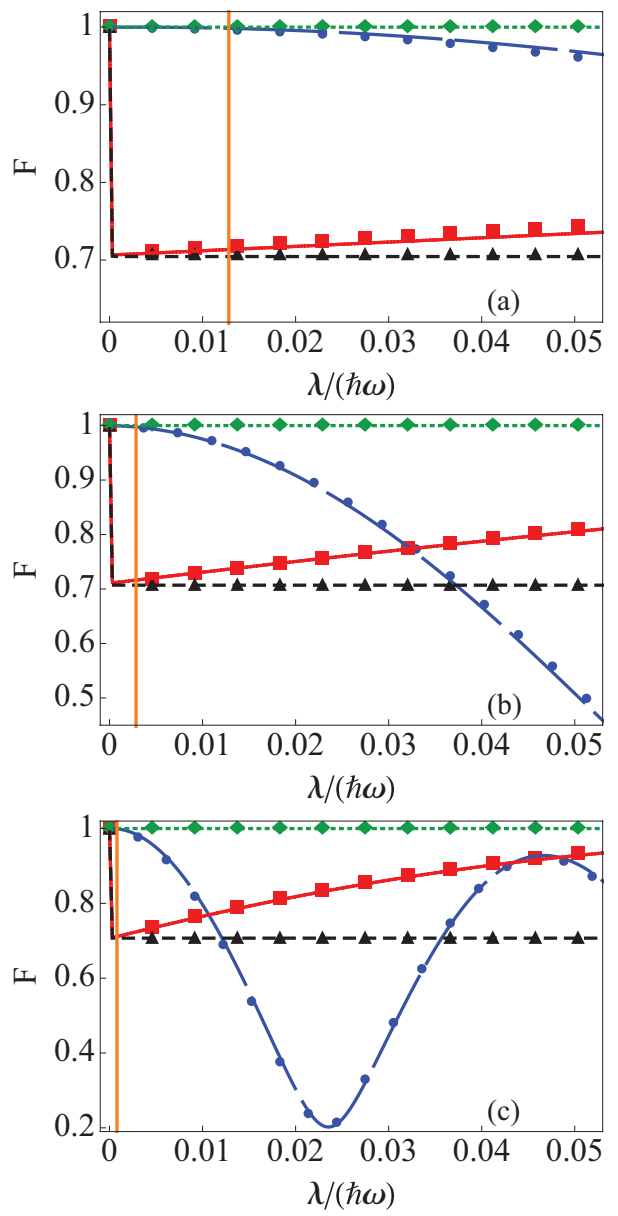

FIG. 2. (Color online) Different fidelities versus the perturbation parameter $\lambda$ for the FF approach (lines) and the two-mode model (symbols). $F_{D}^{(0)}$, long-dashed (blue) line and circles; $F_{D}$, solid (red) line and squares; $F_{S}$, short-dashed (black) line and triangles; $F_{I}$, dotted (green) line and diamonds. The vertical (orange) line is at $0.2 /\left(t_{f} \omega\right)$. (a) $t_{f}=20 \mathrm{~ms}$; (b) $t_{f}=90 \mathrm{~ms}$; (c) $t_{f}=320 \mathrm{~ms}$. $\omega=780 \mathrm{rad} / \mathrm{s}$.

perturbation, typically unknown, due to imperfections of the experimental setting. The adiabatic splitting becomes unstable, as we shall see, but the instability does not depend strongly on this particular form, chosen for simplicity. It would also be found, for example, for a linear-in- $x$ perturbation, a smoothed step, slightly different frequencies for the final right and left traps, or a shifted central barrier [7]. In the final potential configuration, with negligible tunneling, the two wells are independent, and the global ground state is localized in one of them.

To analyze the effects of the perturbation on the wavefunction structure and on the shortcut dynamics, we compute several wave-function overlaps: $F_{S}=\left|\left\langle\psi_{0}^{-}\left(t_{f}\right) \mid \psi_{\lambda}^{-}\left(t_{f}\right)\right\rangle\right|$, the (black) short-dashed line in Fig. 2, is the "structural fidelity" between the (perfectly split) ground state $\psi_{0}^{-}\left(t_{f}\right)$ of the unperturbed potential $V_{\mathrm{FF}}\left(t_{f}\right)$ and the final ground state $\psi_{\lambda}^{-}\left(t_{f}\right)$ of the perturbed potential $V_{\lambda}$. This would be the fidelity found with the desired split state if the process were adiabatic. $F_{S}(\lambda)$ decays extremely rapidly from 1 at $\lambda=0$ to $1 / \sqrt{2}$, which corresponds to the collapse of the ground state of the perturbed potential $V_{\lambda}$ into the deeper well. 
$F_{D}^{(0)}=\left|\left\langle\psi_{0}^{-}\left(t_{f}\right) \mid \psi\left(t_{f}\right)\right\rangle\right|$, the (blue) long-dashed line in Fig. 2, is the fidelity between the state dynamically evolved with $H_{\lambda}, \psi\left(x, t_{f}\right)=\left\langle x\left|e^{i H_{\lambda} t_{f} / \hbar}\right| \psi(0)\right\rangle$, and $\psi_{0}^{-}\left(t_{f}\right) . \psi(0)=$ $\psi_{\lambda}^{-}(0)$ is the initial ground state with $V_{\lambda}(0)$. If $\psi(0)=\psi_{0}^{-}(0)$ is used instead, the results are indistinguishable; see the overlap $F_{I}=\left|\left\langle\psi_{\lambda}^{-}(0) \mid \psi_{0}^{-}(0)\right\rangle\right| \approx 1$ [dotted (green) line] in Fig. 2.

The flat $F_{D}^{(0)}(\lambda)$ at small $\lambda$, in sharp contrast to the rapid decay of $F_{S}(\lambda)$, demonstrates the robustness of the balanced splitting produced by the shortcut. Shorter process times $t_{f}$ make the splitting more and more stable [compare Figs. 2(a)-2(c)]. (We assume condensate lifetimes of the order of seconds; see, e.g., [16].) In principle, $t_{f}$ may be reduced arbitrarily. In practice, this reduction implies an increase in transient energy excitation that requires accurate potential engineering for higher energies [17]. Considering that the time-averaged standard deviation of the energy $\overline{\Delta E}$ should be limited at some value a general bound is $t_{f}>h /(4 \overline{\Delta E})$ [18]. For the trap frequency in the examples $(780 \mathrm{rad} / \mathrm{s})$ and setting $\overline{\Delta E}=\hbar \omega$ the bound saturates for a time $t_{f}=2 \mathrm{~ms}, 10$ times shorter than our shortest time in Fig. 2.

Finally, $F_{D}=\left|\left\langle\psi\left(t_{f}\right) \mid \psi_{\lambda}^{-}\left(t_{f}\right)\right\rangle\right|$ [solid (red) line in Fig. 2] is the fidelity between the dynamically evolved state $\psi\left(t_{f}\right)$ and the final ground state $\psi_{\lambda}^{-}\left(t_{f}\right)$ for the perturbed potential. If the process is adiabatic, then $F_{D} \approx 1$. For very small perturbations $F_{D} \approx F_{S}$. In this regime the dynamical wave function $\psi\left(t_{f}\right)$ is not affected by the perturbation and becomes $\psi_{0}^{-}\left(t_{f}\right)$, up to a phase factor; note that $F_{D}^{(0)} \approx 1$ there. We understand and quantify below this important regime as a sudden process in a moving-frame interaction picture. As $\lambda$ increases, the energies of the ground and excited states of $V_{\lambda}$ separate and the process becomes less sudden and more adiabatic. In Fig. 2(c) for $t_{f}=320 \mathrm{~ms}$ and for large values of $\lambda, F_{D}$ approaches 1 again, and the final evolved state collapses to one side and becomes the ground state of $V_{\lambda}$. For the shorter final times in Figs. 2(a) and 2(b), larger $\lambda$ values are needed so that $F_{D}$ approaches 1 adiabatically.

\section{MOVING TWO-MODE MODEL}

Static two-mode models have been previously used to analyze splitting processes or double-well dynamics $[3,19,20]$. Here we add the separation motion of left and right basis functions to provide analytical estimates and insight. In terms of a (dynamical) orthogonal bare basis $|L(t)\rangle=\left(\begin{array}{l}0 \\ 1\end{array}\right),|R(t)\rangle=$ $\left(\begin{array}{l}1 \\ 0\end{array}\right)$, our two-mode Hamiltonian model is

$$
H(t)=\frac{1}{2}\left(\begin{array}{cc}
\lambda & -\delta(t) \\
-\delta(t) & -\lambda
\end{array}\right)
$$

where $\delta(t)$ is the tunneling rate $[3,19,20]$. We may consider $\lambda$ constant through a given splitting process, for the time being, and equal to the perturbative parameter that defines the asymmetry. A more detailed approach discussed later does not produce any significant difference. The instantaneous eigenvalues are

$$
E_{\lambda}^{ \pm}(t)= \pm \frac{1}{2} \sqrt{\lambda^{2}+\delta^{2}(t)}
$$

and the normalized eigenstates take the form

$$
\begin{aligned}
& \left|\psi_{\lambda}^{+}(t)\right\rangle=\sin \left(\frac{\alpha}{2}\right)|L(t)\rangle-\cos \left(\frac{\alpha}{2}\right)|R(t)\rangle, \\
& \left|\psi_{\lambda}^{-}(t)\right\rangle=\cos \left(\frac{\alpha}{2}\right)|L(t)\rangle+\sin \left(\frac{\alpha}{2}\right)|R(t)\rangle,
\end{aligned}
$$

where $\alpha=\alpha(t)$ is the mixing angle given by $\tan \alpha=\delta(t) / \lambda$.

The bare basis states $\{|L(t)\rangle,|R(t)\rangle\}$ are symmetrical and orthogonal-moving left and right states. Initially they are close to each other and $\delta(0) \gg \lambda$. The instantaneous eigenstates of $H$ are the symmetric ground state $\left|\psi_{0}^{-}(0)\right\rangle=$ $\frac{1}{\sqrt{2}}(|L(0)\rangle+|R(0)\rangle)$ and the antisymmetric excited state $\left|\psi_{0}^{+}(0)\right\rangle=\frac{1}{\sqrt{2}}(|L(0)\rangle-|R(0)\rangle)$ of the single well. At $t_{f}$ we distinguish two extremes:

(1) For $\delta\left(t_{f}\right) \gg \lambda$ the final eigenstates of $H$ tend to symmetric and antisymmetric splitting states $\left|\psi_{\lambda}^{\mp}\left(t_{f}\right)\right\rangle=$ $\frac{1}{\sqrt{2}}\left(\left|L\left(t_{f}\right)\right\rangle \pm\left|R\left(t_{f}\right)\right\rangle\right)$.

(2) For $\delta\left(t_{f}\right) \ll \lambda$ the final eigenfunctions of $H$ collapse and become right- and left-localized states: $\left|\psi_{\lambda}^{-}\left(t_{f}\right)\right\rangle=\left|L\left(t_{f}\right)\right\rangle$ and $\left|\psi_{\lambda}^{+}\left(t_{f}\right)\right\rangle=\left|R\left(t_{f}\right)\right\rangle$.

Since $\delta\left(t_{f}\right)$ is set as a small number to avoid tunneling in the final configuration, the transition from one to the other regime explains the sharp drop in $F_{S}$ at small $\lambda \approx \delta\left(t_{f}\right)$.

\section{A. Moving-frame interaction picture}

We define now a moving-frame interaction-picture (IP) wave function $\psi^{A}=A^{\dagger} \psi^{S}$, where $A=\sum_{\beta=L, R}|\beta(t)\rangle\langle\beta(0)|$ and $\psi^{S}$ is the Schrödinger-picture wave function. $\psi^{A}$ obeys $i \hbar \dot{\psi}^{A}=\left(H_{A}-K_{A}\right) \psi^{A}$, with $H_{A}=A^{\dagger} H A$ and $K_{A}=i \hbar A^{\dagger} \dot{A}$, but for real $\langle x \mid R(t)\rangle$ and $\langle x \mid L(t)\rangle$, the symmetry $\langle x \mid R(t)\rangle=$ $\langle-x \mid L(t)\rangle$ makes $K_{A}=0$.

Inverting Eq. (6) the bare states may be written in terms of the ground and first excited states and energies. The two-level model approximates the actual dynamics by first identifying $\left|\psi_{0}^{ \pm}(t)\right\rangle$ and $E_{0}^{ \pm}(t)$ with the instantaneous ground and excited states and energies of the unperturbed FF Hamiltonian. ${ }^{1}$ We combine them to compute the bare basis in coordinate representation and then the matrix elements $\left\langle\beta^{\prime}\left|H_{\lambda}\right| \beta\right\rangle=$ $H_{\lambda}^{\beta^{\prime} \beta}$, for $\beta \neq \beta^{\prime}$. From Eq. (4), $\delta(t)=-2 H_{\lambda}^{R L}=-2 H_{\lambda}^{L R}$. Once all matrix elements are set we solve the dynamics in the moving frame for the two-mode Hamiltonian. The initial state may be the ground state of the perturbed or unperturbed initial potential. The agreement with the exact results is excellent (see symbols in Fig. 2), which denotes the absence of higher excited states. This two-level model thus provides a powerful interpretative and control tool. To gain more insight we now perform further approximations.

\footnotetext{
${ }^{1}$ Contrast this with the variational approach in [21].

${ }^{2}$ For $\beta=\beta^{\prime}$, we may consistently calculate $\lambda^{\prime}(t):=2\left(H_{\lambda}^{R R}-V_{0}\right)=$ $-2\left(H_{\lambda}^{L L}-V_{0}\right)$, where $V_{0}=\left[E_{\lambda}^{-}(t)+E_{\lambda}^{+}(t)\right] / 2$ is a shift to match the zero-energy point between the FF and the two-mode models. $\lambda^{\prime}$ differs slightly from the constant $\lambda$ at short times, but the results of substituting $\lambda$ with $\lambda^{\prime}$ are hardly distinguishable in the calculations, so the treatment with $\lambda$ is preferred for simplicity.
} 


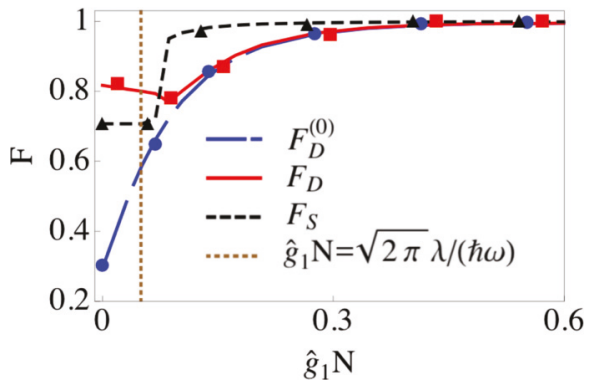

FIG. 3. (Color online) Fidelities vs dimensionless coupling constant for $\lambda /(\hbar \omega)=0.02, t_{f}=320 \mathrm{~ms}$, and $x_{f}=4 \mu \mathrm{m}$. Lines are the same as in Fig. 2. Symbols are for a two-level model like Eq. (4) with the nonlinear diagonal terms $g_{2}\left|c_{R, L}\right|^{2}$ added, where $g_{2}=g_{1} \int d x|R(x)|^{4}=g_{1} \int d x|L(x)|^{4}$ and $\left|c_{R, L}\right|^{2}$ are populations for left and right states [24]. The vertical line is at $\widehat{g_{1}} N=\sqrt{2 \pi} \lambda / \hbar \omega$; see the Appendix.

\section{B. Sudden and adiabatic approximations}

The fidelities at low $\lambda$ may be understood with the sudden approximation in the interaction picture. Its validity requires [22]

$$
t_{f} \ll \hbar / \Delta \overline{H_{A}},
$$

where $\quad \Delta \overline{H_{A}}=\left[\left\langle\psi(0)\left|{\overline{H_{A}}}^{2}\right| \psi(0)\right\rangle-\left\langle\psi(0)\left|\overline{H_{A}}\right| \psi(0)\right\rangle^{2}\right]^{1 / 2}$. We take $|\psi(0)\rangle=\left|\psi_{0}^{-}(0)\right\rangle$ and $\overline{H_{A}}=\frac{1}{t_{f}} \int_{0}^{t_{f}} d t^{\prime} H_{A}\left(t^{\prime}\right)$, where the matrix elements of $H_{A}\left(t^{\prime}\right)$ in the basis $\{|\beta(0)\rangle\}$ coincide with the matrix elements of $H$ in Eq. (4), when the latter are expressed in the basis $\left\{\left|\beta\left(t^{\prime}\right)\right\rangle\right\}$. The condition for the sudden approximation to hold becomes

$$
\lambda \ll \frac{2 \hbar}{t_{f}} .
$$

Vertical lines mark $\lambda=0.2 \hbar / t_{f}$ in Fig. 2 and demonstrate that indeed this condition sets the range in which $F_{D}^{(0)} \approx 1$ so that the fast protocol provides balanced splitting in spite of the asymmetry.

The increase in $F_{D}$ with increasing $\lambda$ can be explained using the adiabatic approximation. The adiabaticity condition is here [23]

$$
\left|\left\langle\psi_{\lambda}^{-}(t) \mid \partial_{t} \psi_{\lambda}^{+}(t)\right\rangle\right| \ll \frac{1}{\hbar}\left|E_{\lambda}^{-}(t)-E_{\lambda}^{+}(t)\right|,
$$

which, using Eqs. (5) and (6), takes the form

$$
\left|\hbar \lambda \dot{\delta}(t) /\left\{2\left[\lambda^{2}+\delta(t)^{2}\right]^{3 / 2}\right\}\right| \ll 1 .
$$

\section{INTERACTING BOSE-EINSTEIN CONDENSATES}

We now generalize the results of the two previous sections for a condensate with interatomic interaction in the mean-field framework. We calculate the ground states $\chi_{N}(x)$ and $\chi_{\frac{N}{2}}(x)$ of a harmonic trap that holds a BEC with $N$ and $N / 2$ atoms and define $f(x, t)=[1-\mathcal{R}(t)] \chi_{N}(x)+\mathcal{R}(t) \chi_{\frac{N}{2}}(x)$, where $\mathcal{R}(t)=3\left(t / t_{f}\right)^{2}-2\left(t / t_{f}\right)^{3} \cdot r(x, t)$ is constructed as

$$
r(x, t)=\left\{f\left[x-x_{0}(t), t\right]+f\left[x+x_{0}(t), t\right]\right\} / z(t),
$$

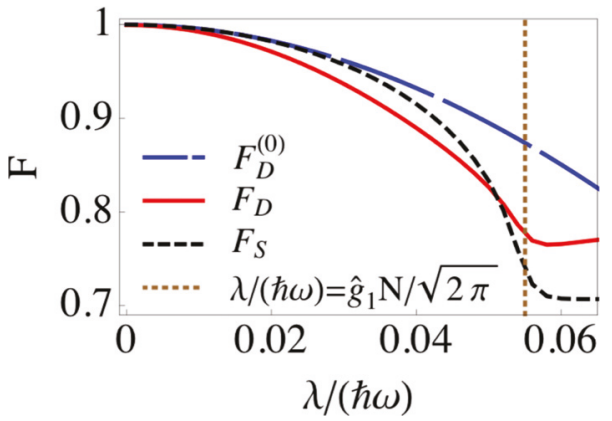

FIG. 4. (Color online) Fidelities for a Bose-Einstein condensate; lines are the same as in Fig. 2. Equation (11) is used to design the potential $V_{\mathrm{FF}}$. Parameters: $x_{f}=4 \mu \mathrm{m}, \omega=780 \mathrm{rad} / \mathrm{s}, \widehat{g_{1}} N=0.138$, and $t_{f}=45 \mathrm{~ms}$.

where $z(t)$ is a normalization factor and $x_{0}(t)=x_{f} \mathcal{R}(t)$. We then get $V_{\mathrm{FF}}$ from Eq. (2) and evolve the initial ground state with the GP equation using the perturbed potential $V_{\lambda}(t)$.

The fidelities are shown in Fig. 3 versus the dimensionless coupling constant $\widehat{g_{1}} N=g_{1} N /\left(\hbar \omega a_{0}\right)$. Note the stabilization of $F_{D}^{(0)}$ towards 1 upon increasing the interaction (this implies more stable shortcuts). $F_{D}$ increases too, as the dynamics tends to be more adiabatic. The structural fidelity jumps to 1 around $\widehat{g_{1}} N=\sqrt{2 \pi} \lambda / \hbar \omega$ from the linear case value $1 / \sqrt{2}$, i.e., balanced splitting by adiabatic following is robust versus trap asymmetry for $\widehat{g_{1}} N \gg \lambda / \hbar \omega$ (see the Appendix). The extra filling of the lower well increases the nonlinear interaction there opposing the external potential imbalance.

The two-level model may also be extended to interacting condensates with minor modifications, also providing an accurate description (see Fig. 3). Adiabaticity fails eventually with decreasing $t_{f}$ and/or $g_{1}$, but the shortcut then provides balanced splitting (see the example in Fig. 4): for small $\lambda$, adiabatic following would be stable (see $F_{S}$ and compare to the sharp drop in Fig. 2 for linear dynamics), but the process is not quite adiabatic $\left(F_{D}<F_{S}\right)$ for the chosen time, $t_{f}=45 \mathrm{~ms}$ - more time would be needed. The shortcut is nevertheless more stable than the hypothetical adiabatic process $\left(F_{D}^{(0)}>F_{S}\right)$.

\section{DISCUSSION}

We have designed simple $Y$-shaped (position- and timedependent) potential traps to fully split noninteracting matter waves rapidly without final excitation, avoiding the instability of the adiabatic approach with respect to slight trap asymmetries. We also avoid or mitigate in this manner the decoherence and noise that affect slow adiabatic following [8,9]. The bifurcation may be experimentally implemented with optical traps created with the aid of spatial light modulators [25]. A simpler approximate approach would involve two Gaussian beams. Further manipulations, such as the application of differential ac Stark phase shifts could be combined with the proposed technique [8]. Also, a differential phase among the two final wave parts will develop due to the imbalance, allowing for precision metrology $[15,16]$, without the time limitations of methods based on adiabatic splitting [16]. In addition, optimal control methods [1-3] complement the 
present approach to further improve stability and/or optimize other variables such as the transient excitation.

A unique feature of the above application of shortcuts to adiabaticity, compared to previous ones [10,26-28], is that the shortcut does not attempt to reproduce the result of an adiabatic following of the perturbed, asymmetrical system in a shorter time. (The assumption has been made so far that the perturbation is uncontrolled and, possibly, unknown.) Instead, the shortcut reproduces the balanced splitting of the adiabatic following corresponding to the unperturbed, perfectly symmetrical system. In other words, shortening the time here is not really the goal, but a means to achieve stability.

Other operations may actually make positive use of the instability due to potential asymmetries. In particular, the ground- and first-excited-state components of the initial trap could be spatially separated by a controlled, slightly asymmetrical adiabatic bifurcation. Moreover, both states would become ground states of the right and left final traps, so the process may as well be used as a population inversion protocol from the excited to the ground state.

We have also analyzed and exemplified the effect of interatomic interaction for a condensate in the mean-field regime. The interaction changes the system behavior with respect to asymmetry, stabilizing dramatically balanced splitting. The total adiabatic collapse of the wave onto one of the two final, separated wells requires, in this case, a significant perturbation, proportional to the coupling constant. Compared to the noninteracting case, this offers different manipulation opportunities, in particular, the possibility of considering the asymmetric perturbation as a known, controllable parameter, so that the imbalance between the two wells may be prepared at will. Examples of this type of manipulation may be found in [29-31]. Shortcuts to adiabaticity and, in particular, the FF approach may be readapted to that scenario by designing the fast protocol taking into account the known, controlled asymmetry. The emphasis would be again, as in most applications of the shortcuts, on accelerating and reproducing the result of a slow process.

Shortcuts to adiabaticity could play other roles in systems described by a double-well with varying parameters. They have been applied, in particular, to speed up the generation of spin-squeezed many-body states in bosonic Josephson junctions [32]. Here we suggest other applications: for example, Stickney and Zozulya [33] have described a wave-function recombination instability due to the weak nonlinearity of the condensate. Specifically, they consider an initially weak ground symmetric mode of the double-well which is exponentially amplified at the expense of an initially strong excited asymmetric mode when the wells are recombined. Similarly to the instability due to asymmetry described in this paper for noninteracting waves, the nonlinear instability is in fact enhanced by adiabatic following. A shortcut-to-adiabaticity strategy such as the one in this paper would stabilize the recombination. Our present results may as well be applied to design $Y$ junctions in planar optical waveguides [34-36], since the equation that describes the field in the paraxial approximation is formally identical to the linear Schrödinger equation, with the longitudinal coordinate playing the role of time. Finally, partial splitting, in which the final two wells are not completely separated and tunneling is still allowed, may as well be considered.

\section{ACKNOWLEDGMENTS}

We are grateful to A. Aspect, B. Juliá-Díaz, J. Martorell, D. Meschede, J. Mompart, and A. Polls for discussions. We acknowledge funding by Projects No. IT472-10, No. FIS2009-12773-C02-01, and No. FIS2012-36673-C03-01 and the UPV/EHU program UFI 11/55. X.C. thanks the National Natural Science Foundation of China (Grant No. 61176118) and the Shanghai Rising-Star Program (Grant No. 12QH1400800). E.T. and S.M.-G. acknowledge support through UPV/EHU fellowships.

\section{APPENDIX: INTERACTION VERSUS ASYMMETRY FOR ADIABATIC FOLLOWING}

Making some simplifying assumptions, we find the conditions under which the interacting condensate ground state splits adiabatically, instead of collapsing into the deepest well. We consider complete splitting of the trap into separated wells and also $\delta\left(t_{f}\right) \ll \lambda$, so that the noninteracting wave would collapse (see Sec. IV). In atomic interferometry, the two split branches of the condensate have to be individually addressed and manipulated during the differential phase accumulation stage, so that tunneling must be negligible $[4,8,37]$. We also assume that the two ground states of the final wells can be approximated by ground states of harmonic oscillators at $\pm x_{f}$, with the right one lifted by $\lambda$ :

$$
\begin{gathered}
V_{L}=\frac{1}{2} m \omega^{2}\left(x+x_{f}\right)^{2}, \\
V_{R}=\frac{1}{2} m \omega^{2}\left(x-x_{f}\right)^{2}+\lambda .
\end{gathered}
$$

The total energy is approximated as $E_{\text {tot }}=E_{L}+E_{R}$. For $j=L, R$,

$$
E_{j}=N_{j} \int d x \phi_{j}\left[-\frac{\hbar^{2} \partial_{x}^{2}}{2 m}+V_{j}\right] \phi_{j}+\frac{1}{2} g_{1} N_{j}^{2} \int d x\left|\phi_{j}\right|^{4},
$$

where $\phi_{j}(x)=\frac{1}{\left[\sqrt{\pi} a_{0}\right]^{1 / 2}} \exp \left[-\left(x \pm x_{f}\right)^{2} / 2 a_{0}^{2}\right]$, and the total number of particles is $N=N_{R}+N_{L}$. The result is

$$
\begin{gathered}
E_{L}=N_{L} \frac{\hbar \omega}{2}+\frac{\widehat{g_{1}}}{2 \sqrt{2 \pi}} \hbar \omega N_{L}^{2}, \\
E_{R}=N_{R}\left(\frac{\hbar \omega}{2}+\lambda\right)+\frac{\widehat{g_{1}}}{2 \sqrt{2 \pi}} \hbar \omega N_{R}^{2},
\end{gathered}
$$

where

$$
\widehat{g_{1}}=g_{1} /\left(\hbar \omega a_{0}\right)
$$

From the minimum-energy condition, $\partial E_{\text {tot }} / \partial N_{R}=0$, it follows that

$$
\frac{\Delta N}{N}=\sqrt{2 \pi} \frac{\lambda / \hbar \omega}{\widehat{g_{1}} N}
$$




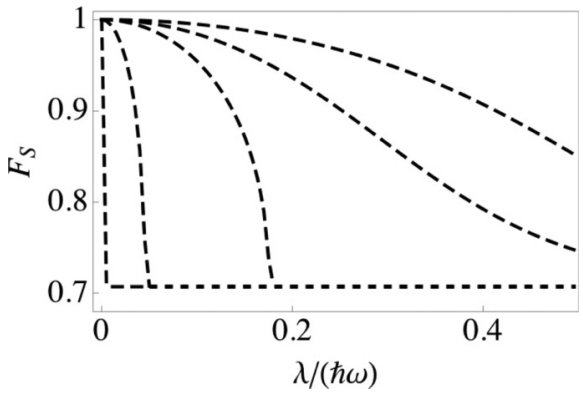

FIG. 5. Structural fidelities for the Bose-Einstein condensate. From left to right, $\widehat{g_{1}} N=0,0.138,0.55,0.69$, and 1.38. In all curves $x_{f}=4 \mu \mathrm{m}$ and $\omega=780 \mathrm{rad} / \mathrm{s}$. Equation (11) was used to design the potential $V_{\mathrm{FF}}$.

with $\Delta N=N_{L}-N_{R}$. (See [16] for a similar treatment in the Thomas-Fermi regime.) Thus, collapse into one well is avoided when $\lambda /\left(\hbar \omega \widehat{g_{1}} N\right) \ll 1$. This relation sets the scale for the uncontrolled and, possibly, unknown asymmetry that may be tolerated to achieve balanced splitting. Adiabatic control of population imbalance requires control of the energy splitting of the order $\lambda \lesssim\left(\hbar \omega \widehat{g_{1}} N\right)$.

Figure 5 shows the structural fidelity $F_{S}(\lambda)$ for several values of $\widehat{g_{1}} N$. The sharp drop at $\widehat{g_{1}} N=0$ is substituted by more and more stable curves as $\widehat{g_{1}} N$ increases. For the splitting described in [29] and [30] using ${ }^{87} \mathrm{Rb}$, we get $\widehat{g_{1}} N \approx 9.5$, quite large compared to the values in Fig. 5. Under these conditions, adiabatic splitting is very stable with respect to minor asymmetries. Moreover, $F_{S}$ decays slowly with respect to $\lambda$, so that the relative population imbalance may be prepared at will by controlling the asymmetry. In [29] and [30] the asymmetry is due to a potential shift that can be controlled with a standard deviation of $100 \mathrm{~nm}$, whereas a displacement of $\sim 1 \mu \mathrm{m}$ is required for the total collapse into one of the wells.
[1] U. Hohenester, P. K. Rekdal, A. Borzi, and J. Schmiedmayer, Phys. Rev. A 75, 023602 (2007).

[2] J. Grond, J. Schmiedmayer, and U. Hohenester, Phys. Rev. A 79, 021603 (2009).

[3] J. Grond, G. von Winckel, J. Schmiedmayer, and U. Hohenester, Phys. Rev. A 80, 053625 (2009).

[4] L. Pezze, A. Smerzi, G. P. Berman, A. R. Bishop, and L. A. Collins, New J. Phys. 7, 85 (2005).

[5] M. Fattori, C. D’Errico, G. Roati, M. Zaccanti, M. Jona-Lasinio, M. Modugno, M. Inguscio, and G. Modugno, Phys. Rev. Lett. 100, 080405 (2008).

[6] M. Gustavsson, E. Haller, M. J. Mark, J. G. Danzl, G. RojasKopeinig, and H. C. Nagerl, Phys. Rev. Lett. 100, 080404 (2008).

[7] J. Gea-Banacloche, Am. J. Phys. 70, 307 (2002).

[8] Y. Shin, M. Saba, T. A. Pasquini, W. Ketterle, D. E. Pritchard, and A. E. Leanhardt, Phys. Rev. Lett. 92, 050405 (2004).

[9] L. A. Collins, L. Pezzé, A. Smerzi, G. P. Berman, and A. R. Bishop, Phys. Rev. A 71, 033628 (2005).

[10] X. Chen, A. Ruschhaupt, S. Schmidt, A. del Campo, D. GuéryOdelin, and J. G. Muga, Phys. Rev. Lett. 104, 063002 (2010).

[11] E. Torrontegui, S. Martínez-Garaot, A. Ruschhaupt, and J. G. Muga, Phys. Rev. A 86, 013601 (2012).

[12] S. Masuda and K. Nakamura, Proc. R. Soc. A 466, 1135 (2010).

[13] M. Demirplak and S. A. Rice, J. Phys. Chem. A 107, 9937 (2003).

[14] S. Masuda and K. Nakamura, Phys. Rev. A 78, 062108 (2008).

[15] T. Schumm et al., Nat. Phys. 1, 57 (2005).

[16] B. V. Hall, S. Whitlock, R. Anderson, P. Hannaford, and A. I. Sidorov, Phys. Rev. Lett. 98, 030402 (2007).

[17] X. Chen and J. G. Muga, Phys. Rev. A 82, 053403 (2010).

[18] J. Anandan and Y. Aharonov, Phys. Rev. Lett. 65, 1697 (1990).

[19] J. Javanainen and M. Y. Ivanov, Phys. Rev. A 60, 2351 (1999).

[20] A. Aichmayr, Bachelor thesis, Institut für Physik Karl-FranzensUniversität Graz (2010).
[21] C. Menotti, J. R. Anglin, J. I. Cirac, and P. Zoller, Phys. Rev. A 63, 023601 (2001).

[22] A. Messiah, Quantum Mechanics, Vol. 2 (Dover, Mineola, NY, 1999).

[23] E. Torrontegui, X. Chen, M. Modugno, A. Ruschhaupt, D. Guéry-Odelin, and J. G. Muga, Phys. Rev. A 85, 033605 (2012).

[24] C. Ottaviani, V. Ahufinger, R. Corbalán, and J. Mompart, Phys. Rev. A 81, 043621 (2010).

[25] V. Boyer, R. M. Godun, G. Smirne, D. Cassettari, C. M. Chandrashekar, A. B. Deb, Z. J. Laczik, and C. J. Foot, Phys. Rev. A 73, 031402(R) (2006).

[26] X. Chen, I. Lizuain, A. Ruschhaupt, D. Guéry-Odelin, and J. G. Muga, Phys. Rev. Lett. 105, 123003 (2010).

[27] Y. Ban, X. Chen, E. Y. Sherman, and J. G. Muga, Phys. Rev. Lett. 109, 206602 (2012).

[28] S. Ibáñez, X. Chen, E. Torrontegui, J. G. Muga, and A. Ruschhaupt, Phys. Rev. Lett. 109, 100403 (2012).

[29] M. Albiez, R. Gati, J. Fölling, S. Hunsmann, M. Cristiani, and M. K. Oberthaler, Phys. Rev. Lett. 95, 010402 (2005).

[30] R. Gati, M. Albiez, J. Fölling, B. Hemmerling, and M. K. Oberthaler, Appl. Phys. B 82, 207 (2006).

[31] J. Estève, C. Gross, A. Weller, S. Giovanazzi, and M. K. Oberthaler, Nature 455, 1216 (2008).

[32] B. Juliá-Díaz, E. Torrontegui, J. Martorell, J. G. Muga, and A. Polls, Phys. Rev. A 86, 063623 (2012).

[33] J. A. Stickney and A. A. Zozulya, Phys. Rev. A 66, 053601 (2002).

[34] A. S. Sanz, J. Campos-Martínez, and S. Miret-Artés, J. Opt. Soc. Am. A 29, 695 (2012).

[35] T.-Y. Lin, F.-C. Hsiao, Y.-W. Jhang, C. Hu, and S.-Y. Tseng, Opt. Express 20, 24085 (2012).

[36] S.-Y. Tseng and X. Chen, Opt. Lett. 37, 5118 (2012).

[37] J. Grond, U. Hohenester, I. Mazets, and J. Schmiedmayer, New J. Phys. 12, 065036 (2010). 